\title{
Caracterización de la ocurrencia e impacto por desastres de origen natural en Panamá. 1990- 2013
}

\section{Carlos Gordón ${ }^{1, *}$}

${ }^{1}$ Investigador asociado, Universidad Santa María La Antigua (USMA), Apartado Postal 0819-08550, Panamá, República de Panamá.

*Autor para correspondencia. Email: carlosg16@gmail.com

Recibido: 13 de noviembre de 2014

Aceptado: 5 de diciembre de 2014

\begin{abstract}
The perception that disasters do not occur in Panama stems largely from the fact that they are identified as small-scale and everyday events. A review of the hydrometeorological and seismic events listed in the database Desinventar for the period 1990- 2013, shows that Panama is a country with high exposure and impacts caused by natural disasters which, for the past 23 years, have affected the lives of half a million people, more than 100,000 houses and caused economic damage of at least US\$ 353.43 million. The perception of a minimum impact associated to natural hazards in Panama has fostered a culture in which development processes do not take into account the inclusion of threats existing in the country. From this perspective, it is necessary to rethink our development model, in order to incorporate effective mechanisms to include the processes of comprehensive disaster risk management into development processes.
\end{abstract}

Keywords: Disasters; Geographic information systems; Disaster risk; Natural hazard; Economic impact.

\section{Resumen}

La percepción de que los desastres no ocurren en Panamá se deriva en gran medida del hecho de que los mismos son identificados como eventos cotidianos y de pequeña escala. Una revisión de los eventos hidrometereológicos y sísmicos recogidos en la base de datos Desinventar para el período 19902013, ha permitido establecer que Panamá es un país con alto grado de exposición e impactos ocasionados por desastres naturales, los cuales durante los últimos 23 años han afectado la vida de medio millón de personas, además de 100 mil viviendas y ocasionado daños económicos por al menos 
US\$353.43 millones. La percepción sobre el impacto reducido de los desastres de origen natural en Panamá ha contribuido a crear una cultura en la que los procesos de desarrollo no toman en cuenta la inclusión de las amenazas existentes en el país. Desde esta perspectiva, se hace necesario replantear nuestro modelo de desarrollo, incorporando mecanismos que hagan efectivos los procesos de gestión integral del riesgo de desastres.

Palabras clave: Desastres; Sistemas de información geográfica; Riesgo de desastres; Amenaza; Impacto económico

\section{Introducción}

Según el Estudio Sitios Importantes para Desastres Naturales realizado por el Banco Mundial, Panamá se encuentra en la posición no 14 entre los países más expuestos a múltiples amenazas, detrás de países como El Salvador (12) y por encima de Nicaragua (15). Panamá tiene un 15\% de su territorio expuesta a desastres y el 12\% de su población vulnerable a dos o más amenazas. Buena parte de esta población expuesta es también la más pobre y la que vive en condiciones más precarias. El crecimiento desordenado, la falta de mecanismos de planificación del desarrollo y el bajo cumplimiento de las regulaciones sobre construcción y uso de suelo son algunos de los factores señalados como agravantes de la vulnerabilidad del país a los desastres (World Bank, 2005, 2012).

No obstante estos datos, en Panamá subsiste la percepción de que somos un país con una baja exposición e impactos por desastres naturales. Esta percepción se deriva en gran medida del hecho de que los desastres que nos afectan, ocurren o son percibidos como eventos cotidianos y de pequeña escala. A pesar de esta percepción, Panamá, tal como lo indica el informe del Banco Mundial, se ve expuesto a una serie de amenazas naturales (Figura 1) entre las que se encuentran:

- Los eventos que mayor impacto ocasionan en Panamá están relacionados con alteraciones de tipo hidrometeorológico. Un régimen de precipitaciones más intensas en lapsos de tiempos cortos, aunado a problemas de degradación de los ecosistemas frágiles que regulan las cuencas y la ocupación y utilización desordenada del territorio, han ocasionado una pérdida de las capacidades regulatorias de los ecosistemas y un aumento de la intensidad de los desastres ocurridos en los últimos años. De los diez eventos con mayores impactos económicos ocurridos entre 2004 y 2013, 9 estuvieron relacionados con tormentas e inundaciones.

- La ocurrencia de sequías en las provincias de Coclé, Veraguas, Herrera y Los Santos, la cual representa un $27 \%$ del país, en esta región se concentra un porcentaje importante de la producción agropecuaria del país. Entre 1982-1983, El Niño Southern Oscillation (ENOS) afectó seriamente la agricultura en esta zona, con pérdidas de US\$14 millones en ganadería y de US\$6 millones en cultivos. Luego, en 1997 -1998, de nuevo este fenómeno produjo pérdidas que alcanzaron US $\$ 40$ millones. Debido al ENOS, el PIB agrícola en ese último periodo se contrajo en 3.7\% (MIDA, 2009). 
Invest. pens. crit.

Vol. 2, No. 5, septiembre-diciembre 2014.

pp. 04-25

\section{Figura 1. Principales amenazas naturales en Panamá}

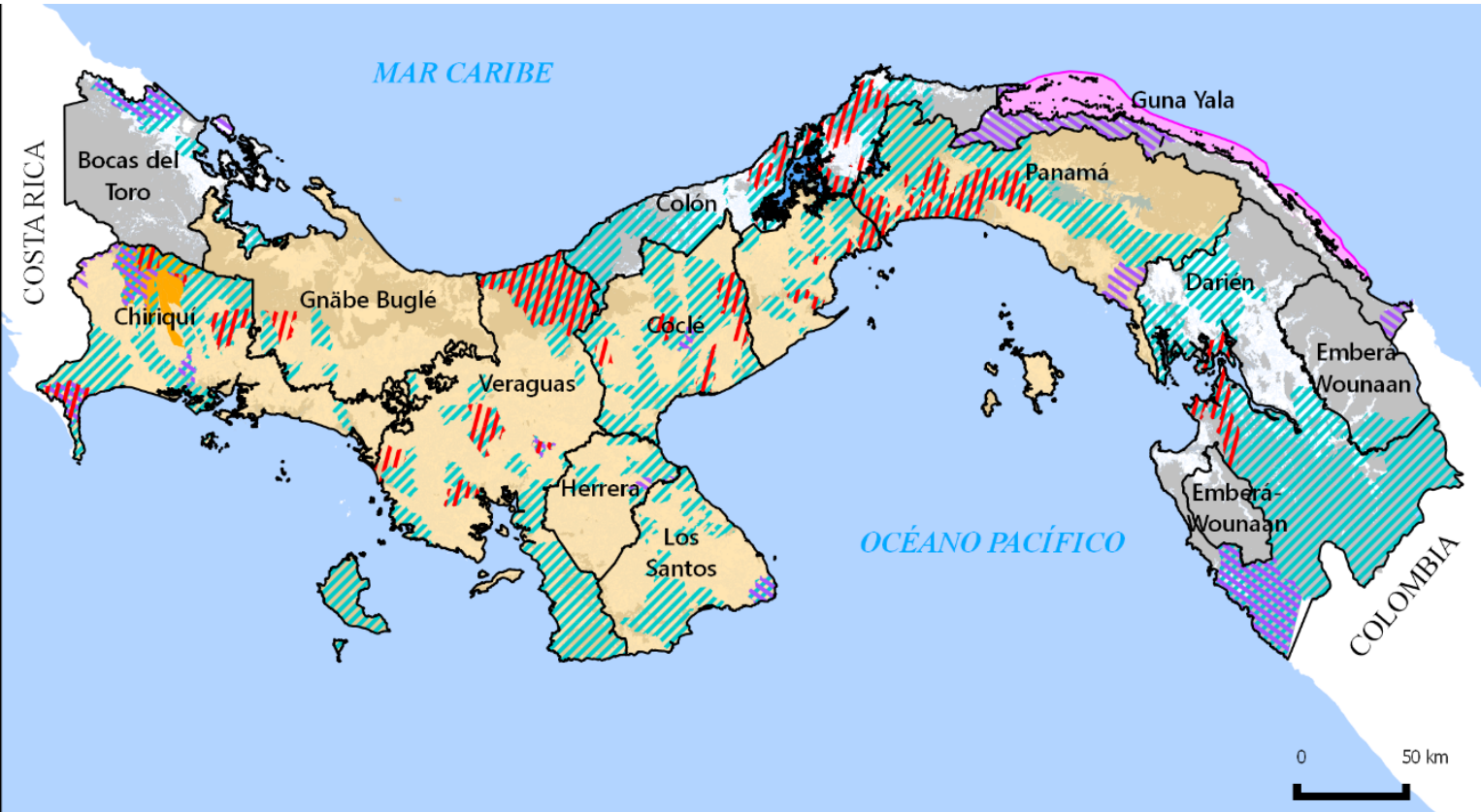

WI/l/. Inundaciones $/ / / / / /$ Deslizamientos $\mathbf{N}$ Sismos $\quad$ Volcán $\quad$ Sequías $\square$ Aumento nivel del mar

Fuente: elaborado por el autor a partir de la base de datos Desinventar; Mapa de zonas de amenaza por lahar en el Volcán Barú, USGS/USAID/SENACYT, 2008; Atlas de las tierras secas y degradadas de Panamá, ANAM, 2008; Climate Change and displacement in the Autonomus Region of Guna Yala, The Peninsula Principles in Action, 2014.

- En cuanto al riesgo sísmico, este se concentra principalmente en la zona occidental, en las provincias de Bocas del Toro y Chiriquí, aunque hay registros de eventos catastróficos en prácticamente todo el país, desde al menos 1621. Los eventos más recientes, de 1991 en Bocas del Toro, y 2002 y 2003 en la provincia de Chiriquí, ocasionaron daños en infraestructura crítica, viviendas, heridos y muertes (DARA/ AECID, 2012). Estudios realizados para la Ciudad de Panamá, han identificado una alta probabilidad de ocurrencia de sismos de magnitud 7 en esta región, lo que podría representar un impacto importante para el desarrollo económico del país dada la alta concentración de activos en esta zona (Rockwell et al, 2010).

- En lo que respecta al riesgo de tsunamis, Panamá tiene registros históricos de la ocurrencia de al menos 3 eventos de este tipo en cada costa —Pacífica y Caribe- siendo el primero en 1621 y el último en 1991, todos relacionados con eventos originados cerca de las costas de Panamá (Instituto de Geociencias, 2013). Una estimación empírica preliminar del riesgo de tsunami indica que el 43\% de los terremotos grandes (mayores a 7 de magnitud superficial $\mathrm{Ms}$ ) a lo largo de la costa del Pacífico de América Central y el 100\% a lo largo del Caribe, podrían generar tsunamis. Terremotos de $7 \mathrm{Ms}$ o mayores con epicentros fuera de la costa o muy cercanos a la costa (en tierra firme) pueden generar tsunamis que podrían impactar estas zonas (Fernández et al, 2000). 
Esta percepción sobre el impacto reducido de los desastres de origen natural en Panamá, ha contribuido a crear una cultura en la que los procesos de desarrollo no toman en cuenta la inclusión de las amenazas existentes (Prensa, 2014). A pesar de que desde 2010, se cuenta con una Política Nacional de Gestión Integral del Riesgo de Desastres, que incorpora las directrices establecidas en el Marco de Acción de Hyogo y de la Política Centroamericana de Gestión de Riesgo de Desastres, aún se carece de instrumentos de gestión del territorio que se traduzcan en acciones concretas que permitan reducir el riesgo de las amenazas a las que se ve expuesto el país.

El riesgo ante las amenazas existentes se ve exacerbado no solo por la ausencia de mecanismos efectivos de ordenamiento territorial y gestión del riesgo de desastres, sino por la condición de acelerado crecimiento económico y de urbanización registrada a partir de la devolución del Canal a administración panameña en el 2000. Durante el período que va de 1950 a 2014, la proporción de población urbana en el país pasó de un 23 a un 75\% con una tasa de crecimiento anual del 3.7\% (Figura 2). El 43\% de la población del país vive en el Área Metropolitana de Panamá (CEPAL, 2014; INEC, 2012). Estos datos reflejan una sostenida tendencia al abandono de las áreas rurales y la concentración de la población en estas áreas urbanas.

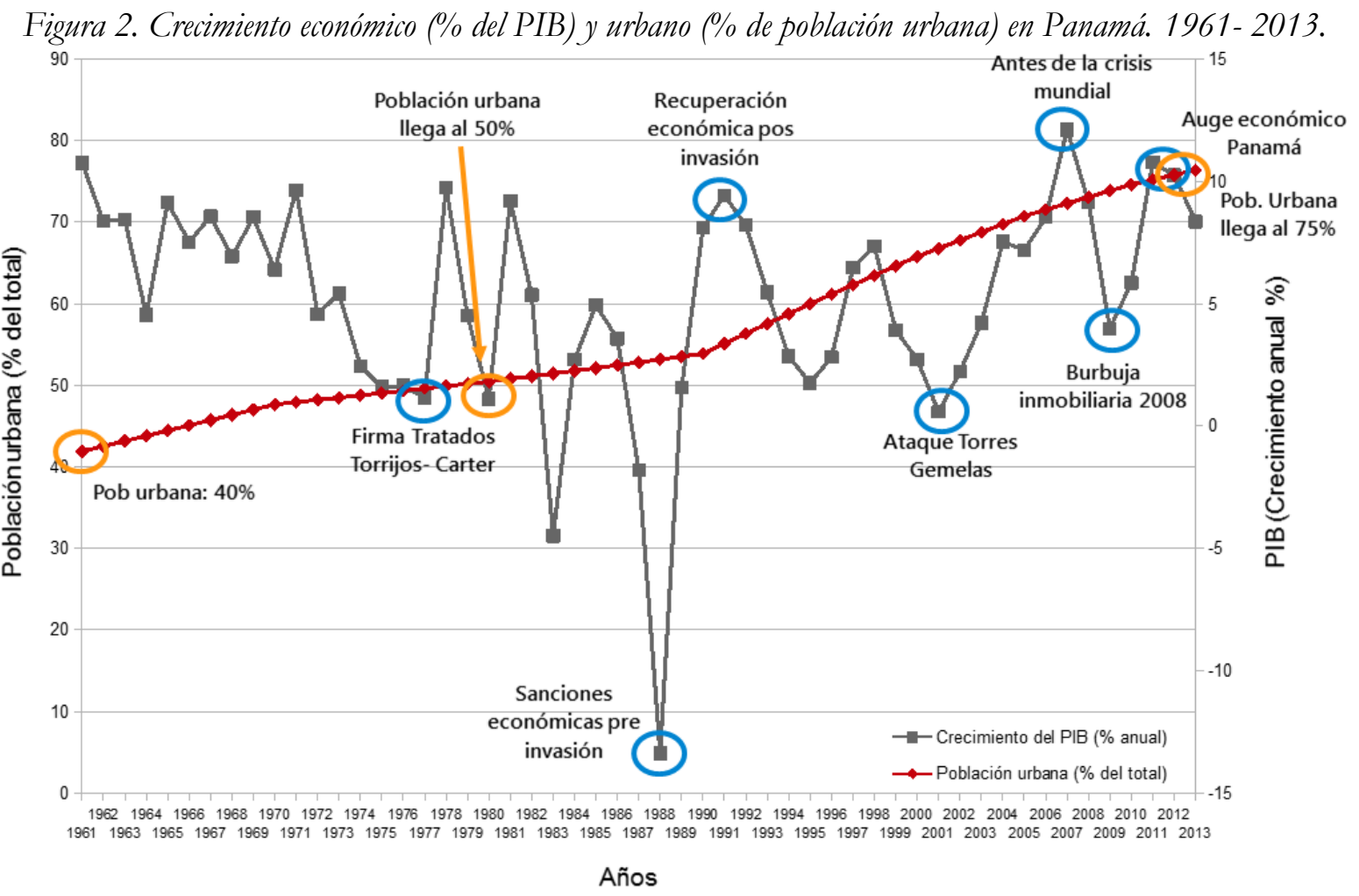

Fuente: elaborado por el autor a partir de la base de datos del Banco Mundial.

En el período 2011 y 2013, la economía panameña ha liderado el crecimiento económico de la región de América Latina y el Caribe, con unas tasas entre el 10 y el 8\% de crecimiento anual (Figura 2). En 
Invest. pens. crit.

Vol. 2, No. 5, septiembre-diciembre 2014.

pp. 04-25

términos absolutos, la economía panameña pasó de US\$20,994 millones de balboas en el 2010 a 42,065 millones en el 2013 (CEPAL, 2014; World Bank, 2014).

Este crecimiento económico se concentra principalmente en las provincias de Panamá y Colón (82.7\% del total del PIB nacional para el 2010) las cuales tienen las mayores rentas per cápita del país (INEC, 2012). Del total de empresas existentes para el 2009, el 39\% se encontraban en el distrito de Panamá y un $5 \%$ en el distrito de San Miguelito. El mayor número de empresas se concentran en actividades de comercio al por mayor, menor y talleres de reparación de vehículos a motor y motocicletas $(11,370)$, seguidas por los hoteles y restaurantes $(3,280)$, otras actividades de servicios $(3,612)$ y la industria manufacturera $(1,950)$ (INEC, 2010).

Las actividades de comercio al por mayor y menor, hotelería y restaurantes y demás concentraban más del 80\% de sus ingresos en los distritos de Panamá y San Miguelito. La industria manufacturera por su parte concentra el 72\% de sus ingresos en estos mismos distritos. El 90\% de los salarios pagados en el sector del comercio al por mayor se concentran en los distritos de Panamá y San Miguelito (INEC, 2011).

Debido a este escenario, de acelerado crecimiento económico y escasos mecanismos de ordenación del desarrollo, se hace urgente cambiar la percepción de que Panamá es un país con un bajo nivel de riesgo y amenazas ante desastres naturales. Para esto, se requiere generar un inventario sistemático de desastres y pérdidas, además de mejorar las capacidades para la identificación del riesgo, ampliando el limitado alcance de las evaluaciones de vulnerabilidad, principalmente realizadas hasta el momento, en el área del Canal (Banco Interamericano de Desarrollo, 2012).

La Ley 7 de 11 de febrero 2005, por la cual se crea el Sistema Nacional de Protección Civil (SINAPROC), establece dentro de sus funciones la responsabilidad de crear y mantener sistemas de información para la gestión de riesgo de desastre. En ese sentido, SINAPROC mantiene el Sistema de Inventario de Desastre DESINVENTAR, que es una base de datos que compila el registro de eventos según tipo e identifica el número de personas y viviendas afectadas para cada uno de estos eventos (Desinventar.org, 2014). No obstante, esta base de datos no reemplaza la necesidad de un sistema de información integral que brinde información sobre las amenazas, vulnerabilidades existentes en el territorio, y los patrones del riesgo del país.

Las referencias sobre la situación de país en el tema de desastres naturales, son relativamente escasas y están centradas hacia el abordaje de temas o eventos específicos como: los informes de país sobre la implementación de la Plataforma de Hyogo (SINAPROC, 2012); estudios sobre la amenaza volcánica, sísmica y tsunamis (US Geological Survey; 2008, Fernández, 2000, 2011; Seismo, 1999; Camacho, 1994), estudios sobre temas de gestión de cuencas y ordenamiento territorial (ANAM, 2014; CONADES, 2014), informes sobre ocurrencia de eventos catastróficos (Espinosa, 2011; Linkimer, 2004; Soulas, 1991), análisis sobre el riesgo y la vulnerabilidad creciente, (Sanahuja, Coles y Froes 2011) y 
sobre los impactos futuros del cambio climático en sectores como la agricultura, los ecosistemas y costas del país (CATHALAC, 2008; Mora et al, 2010; Peninsula Principles in Action, 2014).

Ante este vacío en la sistematización de la información, este trabajo tiene como objetivo, caracterizar la ocurrencia e impacto por desastres naturales en Panamá durante el período 1990- 2013 e identificar las áreas con mayor amenaza y exposición ante estos eventos, con el fin de proveer de insumos que permitan orientar las actividades que realizan tanto instituciones, ciudadanos y organizaciones de la sociedad civil en temas de atención, prevención, reducción y mitigación de los daños ocasionados por desastres de origen natural.

\section{Materiales y métodos}

Para la elaboración de esta caracterización sobre la ocurrencia e impacto por desastres de origen natural en Panamá, se utilizaron las siguientes fuentes:

- Revisión bibliográfica, principalmente en la Gaceta Oficial y en la prensa local para compilar datos sobre los principales desastres ocurridos en Panamá durante el período de estudio.

- Base de datos Desinventar. De esta base de datos se utilizaron las siguientes variables: lugar, corregimiento, distrito y provincia de ocurrencia, fecha, tipo de evento, personas afectadas, muertes y viviendas afectadas. Para el análisis solo se incorporaron los datos correspondientes a eventos hidrometereológicos y geológicos ocurridos entre 1990 a 2013.

- Datos del Censo de Población y Vivienda 2010: se utilizaron las variables de acceso a la vivienda a servicios básicos (electricidad, agua potable, servicio sanitario), materiales de la vivienda (piso, techo y pared), ingreso promedio mensual de la vivienda, densidad de población (hab/ha), migrantes recientes (2006-2010), viviendas construidas recientemente (2005-2010), población indígena viviendas y población total.

- La base cartográfica de red de carreteras y división politica del país a nivel de corregimientos, distritos y provincias actualizada al 2010, proporcionada por el Instituto Nacional de Estadística y Censo.

Con base en estos datos se obtuvieron frecuencias, porcentajes y tasas (letalidad, como resultado de dividir el número de muertes por evento contra la población afectada durante el período) para las variables seleccionadas, las cuales se graficaron y mapearon utilizando los software MS Excel (para generación de gráficos) y QGIS software open source de sistemas de información geográfica, para la generación de mapas.

Con el fin de elaborar un mapa que permitiera reflejar el carácter multiamenaza de Panamá, se procedió a identificar todos aquellos corregimientos que en la base de datos Desinventar, habían registrado algún tipo de desastre durante el período de estudio. Para completar este análisis, se incorporaron aquellas áreas bajo riesgo debido a aumento del nivel del mar, principalmente en el Archipiélago de Guna Yala, (Peninsula Principles in Action, 2014) y por amenaza volcánica del Volcán Barú en Chiriquí (US Geological Survey, 2008). 
Invest. pens. crit.

Vol. 2, No. 5, septiembre-diciembre 2014.

pp. 04-25

Finalmente, se generó un Índice de Riesgo Manifiesto (IRM) a partir de los datos de Desinventar, utilizando el software de minería de datos Rapid Miner (aplicando una normalización de las variables utilizadas y un análisis de componentes principales) y tomando las variables de personas afectadas, muertes registradas y viviendas afectadas según corregimientos en el período 1990- 2013. El índice de riesgo manifiesto (IRm), se puede definir como una combinación algebraica específica de variables del daño incluidas en DesInventar, que nos permite caracterizar una unidad de resolución determinada (provincia, distrito, corregimiento) según su nivel de riesgo manifiesto. De esta manera, se estaría apelando a una metodología híbrida para la evaluación del riesgo, deduciendo patrones de riesgo a partir de evidencia empírica expresada en el daño, e infiriendo distintos niveles del riesgo, a partir de una combinación de las distintas formas en que representamos ese daño (Sanahuja, 1999).

Los resultados de este índice generan una escala, donde los valores menores de la escala indican un menor riesgo manifiesto, mientras que aquellos con valores más altos, indican una mayor manifestación del riesgo.

\section{Resultados}

Datos básicos generales

Durante el período 1990 a 2013, en la República de Panamá se registraron un total de 2,717 eventos de origen natural, de los cuales el $57 \%$ corresponden a inundaciones, $17 \%$ a vendavales/ vientos fuertes y el 15\% a deslizamientos (Figura 3). La mayor parte de estos eventos se concentran en las provincias de Panamá, Chiriquí y Coclé, siendo los corregimientos de Juan Díaz, Las Cumbres y Belisario Porras, todos en la provincia de Panamá, donde se concentra el 7\% de los eventos registrados durante el período de estudio.

Respecto a los impactos de estos eventos, la base de datos Desinventar identifica un total de 529,733 personas afectadas, siendo los eventos con mayores afectados, las inundaciones $(463,864)$, los vendavales/ vientos fuertes $(38,826)$ y las marejadas $(5,101)$. El 16\% del total de personas afectadas $(88,559)$ se concentraron en 5 corregimientos del país, dos de estos, los corregimientos de Juan Díaz y Tocumen, en el Área Metropolitana de Panamá (AMP), concentran el 8\% de todos los afectados.

En Panamá se ha registrado un total de 322 muertes debido a desastres naturales, siendo la principal causa de muertes las inundaciones (138), las tormentas eléctricas (69), los deslizamientos (64), los vendavales (22) y los tornados (18). Los corregimientos que registran el mayor número de defunciones por desastres naturales, corresponden a Pacora, distrito y provincia de Panamá (13), El Potrero en La Pintada, Coclé (13) y Guabito en Changuinola, Bocas del Toro (10). 
Figura 3. Eventos registrados según tipo. Panamá: 1990- 2013
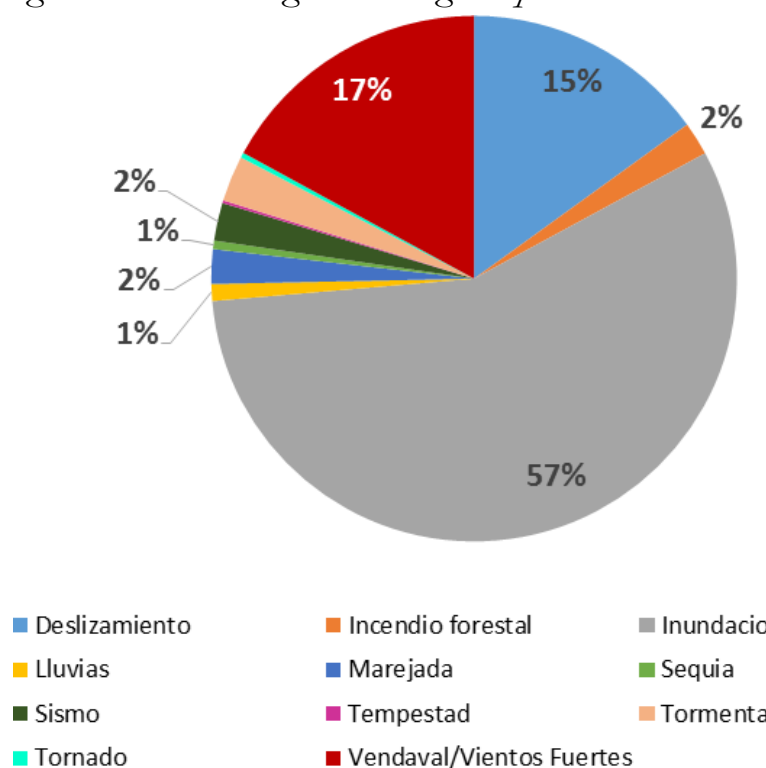

$$
\begin{array}{ll}
\text { Incendio forestal } & \text { Inundacion } \\
\text { Marejada } & \text { Sequia } \\
\text { - Tempestad } & \text { Tormenta electrica } \\
\text { - Vendaval/Vientos Fuertes } &
\end{array}
$$

Fuente: elaborado por el autor a partir de la base de datos de Desinventar

Las afectaciones en el sector viviendas corresponden a un total de 100,731, de las cuales la mayor parte corresponde a inundaciones (87\%), vendavales (8\%), mientras que sismos, deslizamientos, lluvias, vendavales/ vientos fuertes y marejadas, corresponden cada una a un $1 \%$ de todas las viviendas afectadas. Al igual que en el caso de los impactos en la población, las afectaciones en viviendas se concentran en los corregimientos de Juan Díaz en la provincia de Panamá $(6,723)$, Guabito en Bocas del Toro $(3,492)$ y Cativá en Colón $(3,001)$.

El análisis de los impactos en población y viviendas por áreas geográficas permite determinar que el $60 \%$ de los impactos en las viviendas y el $62 \%$ en personas se concentran en las áreas rurales del país, mientras que en el AMP, corresponde al 22 y 20\% respectivamente y en las áreas indígenas al 13 en viviendas y $14 \%$ del total de personas afectadas en el país.

$\mathrm{Al}$ analizar el número de eventos, viviendas y personas afectadas como promedios por semana, se pudo identificar dos ciclos dentro del período de estudio. El primer ciclo iniciaría en 1990 y finalizaría en 2001, mientras que el segundo iniciaría en 2002 y terminaría en el 2013. Al comparar el número de viviendas y personas afectadas entre los dos períodos, vemos que el 59.35\% de las personas y $61 \%$ de las viviendas afectadas por desastres naturales reportadas entre 1990 y 2013 se dieron en el último decenio (2002- 2013). De la misma forma, aumentaron los promedios anuales de personas y viviendas afectadas en los últimos diez años un 1.6 veces respecto a los diez años anteriores (Figura 4). 
Invest. pens. crit.

Vol. 2, No. 5, septiembre-diciembre 2014.

pp. $04-25$

Figura 4. Promedio de personas y viviendas afectadas por desastres naturales por semana. Panamá: 1990- 2013.
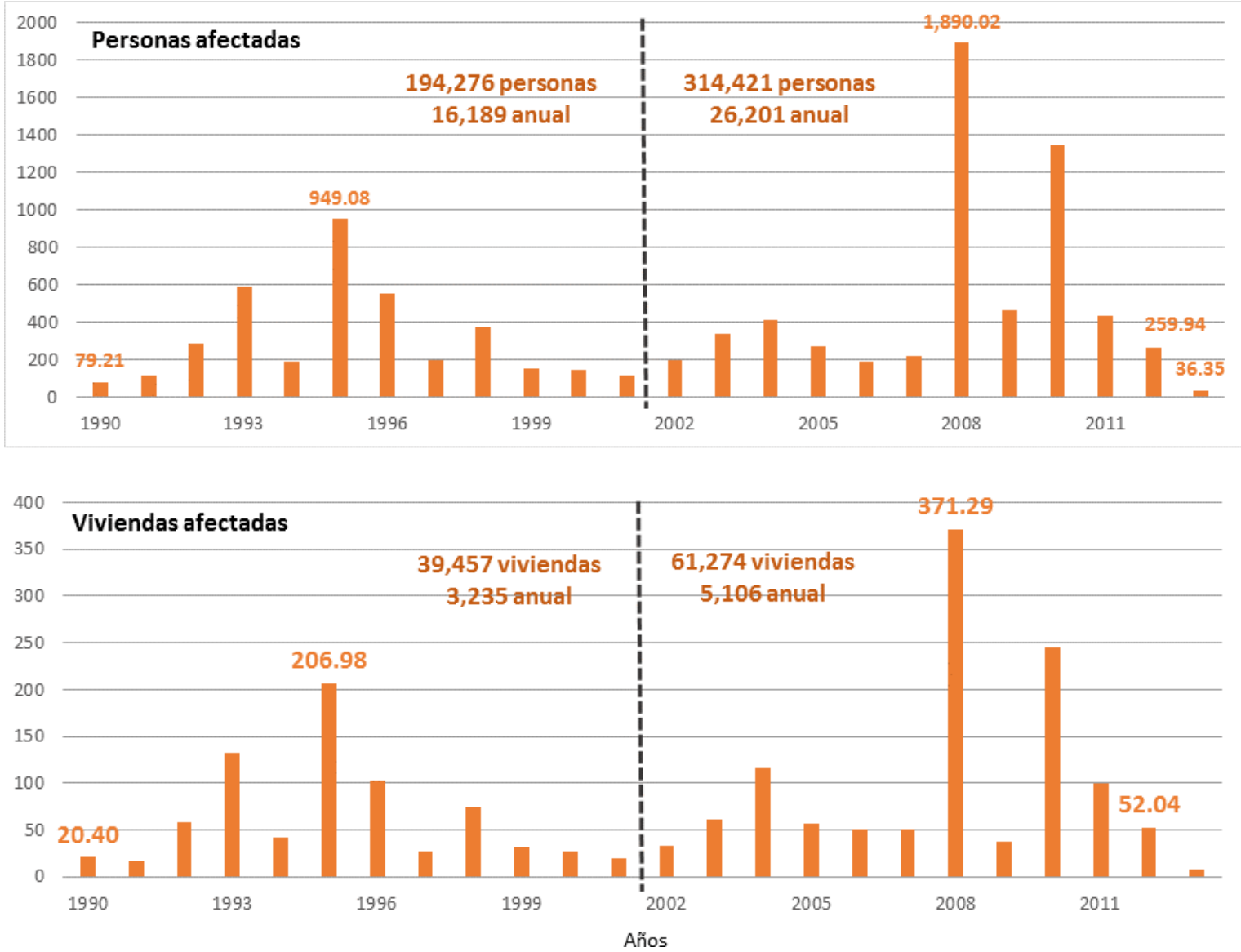

Fuente: elaborado por el autor a partir de la base de datos de Desinventar

Tipologías de eventos: frecuencia e impactos

Con el fin de determinar un perfil de país que permitiese identificar los distintos tipos de amenazas naturales que afectan a la población, se procedió a mapear, tabular y graficar los datos compilados en la base de datos de Desinventar, analizándolos según tipologías de eventos. A continuación se presentan los hallazgos encontrados para cada tipo de evento:

a) Deslizamientos: Muestran una ligera tendencia al aumento, pasando de 30 eventos en 1992, a 36 en el 2011, con un máximo de 290 deslizamientos en el 2008. El mayor número de deslizamientos ocurre en el distrito de San Miguelito, que concentra el 25\%, siendo los corregimientos de Belisario Porras (62) y Amelia Denis de Icaza (20) los que mayor frecuencia registran.

Respecto al número de afectados, la tendencia también es al aumento, pasando de 186 afectados en 1992 a 464 en el 2010, con un número máximo de 577 en el 2001. Belisario Porras en el distrito de San Miguelito (551), Provincia de Panamá, Cerro Punta (517) en Bugaba, Chiriquí y Calovébora (400) en Santa Fe de Veraguas, son los tres corregimientos con el mayor número 
de afectados por deslizamientos, concentrando el 34\% de todos los eventos de este tipo registrados durante el período de estudio. Las muertes por este tipo de desastres se registraron principalmente en el distrito de Portobelo en Colón (16), Belisario Porras en San Miguelito, Panamá, Cerro Punta en Chiriquí y Chiriquí Arriba en el distrito de Penonomé, provincia de Coclé (estos tres últimos con 6 muertes cada uno). El porcentaje de letalidad global de este tipo de eventos es de $1.48 \%$. En cuanto a las viviendas afectadas, el corregimiento que mayores afectaciones concentra, es el corregimiento de Puerto Armuelles, con 178 viviendas, seguido de los corregimientos de Belisario Porras (108) y Calovébora (80).

Tabla 1. Principales indicadores de la ocurrencia e impactos por desastres naturales en Panamá. 1990- 2013

\begin{tabular}{rrrrrr}
\hline Año & Eventos & Personas & Viviendas & Muertes & Letalidad (\%) \\
\hline 1990 & 46 & 6,127 & 1,061 & 11 & 1.80 \\
1991 & 30 & 6,067 & 853 & 10 & 1.65 \\
1992 & 83 & 14,928 & 3,039 & 53 & 3.55 \\
1993 & 82 & 30,775 & 6,841 & 23 & 0.75 \\
1994 & 87 & 9,974 & 2,173 & 25 & 2.51 \\
1995 & 109 & 49,373 & 10,763 & 14 & 0.28 \\
1996 & 144 & 28,643 & 5,370 & 18 & 0.63 \\
1997 & 191 & 11,812 & 1,369 & 6 & 0.51 \\
1998 & 258 & 19,399 & 3,905 & 8 & 0.41 \\
1999 & 105 & 7,749 & 1,612 & 15 & 1.94 \\
2000 & 130 & 7,619 & 1,442 & 0 & 0.00 \\
2001 & 122 & 5,819 & 1,029 & 9 & 1.55 \\
2002 & 51 & 10,322 & 1,737 & 5 & 0.48 \\
2003 & 51 & 17,689 & 3,141 & 5 & 0.28 \\
2004 & 82 & 29,832 & 6,020 & 23 & 0.77 \\
2005 & 72 & 14,805 & 2,913 & 1 & 0.07 \\
2006 & 91 & 12,177 & 2,607 & 10 & 0.82 \\
2007 & 293 & 11,378 & 2,611 & 0 & 0.00 \\
2008 & 219 & 101,715 & 19,307 & 35 & 0.34 \\
2009 & 63 & 23,885 & 1,915 & 10 & 0.42 \\
2010 & 101 & 71,723 & 12,729 & 22 & 0.31 \\
2011 & 113 & 22,515 & 5,152 & 2 & 0.09 \\
2012 & 124 & 13,517 & 2,706 & 17 & 1.26 \\
2013 & 70 & 1,890 & 436 & 0 & 0.00 \\
\hline TOTAL & $\mathbf{2 , 7 1 7}$ & $\mathbf{5 2 9 , 7 3 3}$ & $\mathbf{1 0 0 , 7 3 1}$ & $\mathbf{3 2 2}$ & $\mathbf{0 . 6 1}$ \\
\hline
\end{tabular}

Fuente: elaborado por el autor a partir de la base de datos de Desinventar

b) Incendios forestales: Un total de 57 eventos se registraron en el período de estudio, siendo el año con el mayor número de incendios 1998 (24), y registrándose un promedio de 2.5 eventos al 
Invest. pens. crit.

Vol. 2, No. 5, septiembre-diciembre 2014.

pp. 04-25

año. El incendio forestal con el mayor número de personas afectadas se dio en 1992 (300 personas y 60 viviendas), concentrando el 75\% del total de afectados, el mismo se registró en la provincia de Veraguas, aunque no se puede determinar cuáles fueron los corregimientos o distritos involucrados. En cuanto a las muertes, solo se registraron dos defunciones por este tipo de eventos en el período de estudio. El porcentaje de letalidad global de los incendios forestales es de $0.51 \%$.

c) Inundaciones: Las inundaciones son los eventos que mayor frecuencia e impacto tienen en $\mathrm{Pa}$ namá. Durante el período de estudio la tendencia ha sido al aumento, pasando de 16 inundaciones en 1990 a 35 en el 2013, con un máximo de 230 en el 2008, y un promedio de 66 eventos al año. El corregimiento de Juan Díaz (Panamá) es el registra el mayor número de inundaciones, con 56, seguido de Las Cumbres (Panamá), Tonosí (Los Santos) y Pacora (Panamá).

Respecto al número de afectados, también se ha registrado un incremento, pasando de 3,713 personas en 1990 a 12,370 en el 2012, llegando a 100,713 en el 2008, siendo este último año, el que mayor número de muertes registró (20). Los corregimientos con el mayor número de afectados corresponden a Juan Díaz (Panamá, 30,552), Cativa en Colón $(13,853)$ y Soná en Veraguas $(11,181)$. Para las inundaciones el porcentaje de letalidad global es de $0.03 \%$.

El número de viviendas afectadas por inundaciones también ha registrado un incremento, pasando de 655 viviendas afectadas en 1990, a 2475 en 2012, con un máximo de 18,931 en 2008. En cuanto a los corregimientos con mayores afectaciones, los daños en las viviendas se concentran en Juan Díaz, Panamá (6,608), Cativá en Colón (2,954) y Tocumen (2,336) en Panamá.

d) Lluvias: La base de datos registra unos 30 eventos de lluvia, un promedio de 1.3 eventos al año. A pesar de su baja frecuencia, los impactos de estos eventos son importantes, afectando a unas 4,345 personas, 902 viviendas y 2 personas fallecidas. El mayor número de eventos de lluvia registrados se da en el corregimiento de 24 de diciembre, provincia y distrito de Panamá. El porcentaje de letalidad de estos eventos es del $0.05 \%$.

e) Marejadas: Representan el 2.2\% de los eventos registrados, 19 de los 60 eventos registrados se dieron en 1997, dándose el mayor número de eventos en el corregimiento de Puerto Caimito, distrito de La Chorrera, Panamá. Las marejadas han afectado un total de 5,101 personas, el $29 \%$ de estas en el corregimiento de Puerto Caimito. Por este tipo de eventos se registran 6 personas fallecidas. La letalidad de las marejadas es del $0.12 \%$. Unas 846 viviendas se han visto afectadas por marejadas durante el período de estudio.

f) Sequías: para el caso de las sequías la base de datos solo registra 15 eventos, todos para el año 1997, esto a pesar de que por ejemplo, entre enero y abril de 2013, y en mayo- julio de 2012, el Gobierno de Panamá se vio obligado a realizar declaraciones de emergencia debido a estos eventos. De esta forma, se puede indicar entonces, que para el caso de las sequías la base de 
datos de Desinventar presenta un grado significativo, aunque no cuantificado en este estudio, de subregistro.

g) Sismos: Los sismos representan el 2.4\% de los eventos registrados (67 eventos). El mayor número de sismos registrados en la base de datos se dio en 2000, con 12. El mayor número de personas afectadas se dio en el evento de Puerto Armuelles, en Chiriquí en el 2003, con un total de 7,743 personas y 1,616 viviendas. La base de datos Desinventar no presenta datos de las afectaciones ocurridas por el sismo de abril de 1991 (el sismo más importante ocurrido en los últimos 23 años en Panamá), y del cual se reportan unas 25 personas muertas, 5,450 personas, 1,700 viviendas afectadas y daños por unos US\$100 millones de dólares (Soulas, 1991). En su conjunto, los sismos registraron una letalidad de $0.02 \%$.

b) Tempestad: La base de datos registra solo 5 tempestades, 4 en 1996 y 1 en 1997, con un total de 177 personas y 33 viviendas afectadas. No se registran muertes por esta causa.

i) Tormenta eléctrica: De 77 tormentas eléctricas registradas el 87\% se concentran en la década de 1990, siendo el año con el mayor número 1996 (12). Las tormentas eléctricas han afectado un total de 428 personas, 43 viviendas y están relacionadas con 69 fallecimientos, siendo la amenaza natural que mayor número de muertes registra, detrás de las inundaciones (138). El mayor número de muertes se concentra en las provincias de Panamá y Veraguas. Las tormentas eléctricas es el evento de mayor letalidad registrada con un $16 \%$.

j) Tornados: Los tornados son los eventos de menor frecuencia registrada en Panamá, con un total de 8. A pesar de esto, concentran un número significativo de daños en viviendas (814), personas $(4,076)$ y muertes $(18)$, estas últimas correspondientes a un mismo evento que afectó los corregimientos de Juan Díaz, Pedregal, Tocumen y Río Abajo en ciudad de Panamá en 1992. En el 2009, otro evento reportado en el distrito de San Miguelito, afectó unas 700 personas y 152 viviendas, sin que se registraran muertes. La letalidad de estos eventos es de $0.44 \%$.

k) Vendavales / vientos fuertes: Los vendavales representan el 17.4\% del total de eventos registrados. Los mismos se presentan con una frecuencia de 20.5 eventos al año, y durante el período de estudio han mostrado una tendencia a mantenerse estables, con un máximo de eventos en 1997 (65). El mayor número de vendavales ocurren en los corregimientos de Arraiján en la provincia de Panamá (13), David, Chiriquí (12) y Penonomé, Coclé (10).

En cuanto al número de afectados, los vendavales registran unas 38,826 personas afectadas $(7.3 \%)$, con una tendencia mantenerse estable en un promedio de 1,688 personas al año, con un pico en 1993, cuando se registraron 19,138 personas afectadas. El mayor número de afectados por vendavales se concentra en los corregimientos de Guabito en Bocas del Toro (14,074), La Mina $(1,981)$ en Herrera y Belisario Porras $(1,069)$ en Panamá. 
Invest. pens. crit.

Vol. 2, No. 5, septiembre-diciembre 2014.

pp. $04-25$

Un total de 7,811 viviendas se han visto afectadas por vendavales en Panamá, de estas el mayor número se dio en los años 1992 (1,164) y 1993 (3,657), siendo el promedio anual de viviendas afectadas de 339. Espacialmente los daños en viviendas debido a los vendavales se concentran en Guabito en Bocas del Toro (2,814), La Mina (396) en Herrera y Belisario Porras (215) en Panamá.

Las muertes relacionadas con vendavales representan el 6.5\% del total de muertes por desastres registradas en el país, siendo el porcentaje de letalidad un $0.05 \%$.

Evaluación de la amenaza, la exposición y el riesgo

Con el fin de poder dimensionar de forma aproximada las áreas expuestas a un mayor número de amenazas y determinar cuánto de la población, superficie y bienes del país están expuestos a los mismos, se elaboraron dos mapas (Figura 5), que muestran, por un lado, la severidad del daño ocasionado por los desastres registrados hasta la fecha (índice de riesgo manifiesto), y por el otro, la cuantificación de los elementos expuestos al daño por estas amenazas naturales (mapa de exposición multiamenaza).

Tabla 2. Exposición de bienes y población según número de amenazas existentes en cada corregimiento de Panamá.

\begin{tabular}{lrrrrrrrr}
\hline & \multicolumn{7}{c}{ NO DE AMENAZAS EN CADA CORREGIMIENTO } & T \\
\cline { 2 - 7 } EXPOSICIÓN & $\mathbf{0}$ & $\mathbf{1}$ & $\mathbf{2}$ & $\mathbf{3}$ & $\mathbf{4}$ & $\mathbf{5}$ & $\mathbf{6}$ & TOTAL \\
\hline Población & 853,543 & 374,826 & 452,836 & 926,967 & 494,537 & 168,677 & 134,427 & $3,405,813$ \\
Población (\%) & 25.06 & 11.01 & 13.30 & 27.22 & 14.52 & 4.95 & 3.95 & 100.00 \\
Superficie (has) & $3,361,709.98$ & $1,801,184.74$ & $1,055,088.04$ & $1,011,125.11$ & $130,362.68$ & $47,466.57$ & $33,232.10$ & $7,440,169.21$ \\
Superficie (\%) & 45.18 & 24.21 & 14.18 & 13.59 & 1.75 & 0.64 & 0.45 & 100.00 \\
Viviendas & 210,018 & 100,826 & 121,819 & 248,150 & 132,223 & 45,593 & 37,439 & 896,068 \\
Viviendas (\%) & 23.44 & 11.25 & 13.59 & 27.69 & 14.76 & 5.09 & 4.18 & 100.00 \\
Empresas & 8,526 & 6,790 & 9,118 & 21,990 & 10,529 & 2,898 & 5,474 & 65,325 \\
Empresas (\%) & 13.05 & 10.39 & 13.96 & 33.66 & 16.12 & 4.44 & 8.38 & 100.00 \\
Carreteras (km) & $26,679.33$ & $14,039.88$ & $10,534.12$ & $8,929.12$ & $2,866.76$ & $1,451.98$ & $1,004.74$ & $65,505.93$ \\
Carreteras (\%) & 40.73 & 21.43 & 16.08 & 13.63 & 4.38 & 2.22 & 1.53 & 100.00 \\
\hline
\end{tabular}

Fuente: elaborado por el autor a partir de la base de datos de Desinventar, Censo de Población y Vivienda, Directorio de Establecimientos y cartografía de la red vial del INEC

a) Mapa de exposición multiamenaza: A partir de estos datos se pudo determinar que son los corregimientos de Puerto Armuelles, Boquete, Volcán y David en Chiriquí, Santiago y La Mesa en Veraguas, Antón en Coclé y Juan Díaz, Pueblo Nuevo y Arraiján en el Área Metropolitana de Panamá, los corregimientos que registran un mayor número de amenazas por desastres de origen natural (de entre 4 a 6 amenazas).

El 54\% del territorio del país y el 75\% de la población se ve expuesto a al menos una amenaza por desastres naturales. El 9\% de la población y el 2\% del territorio se ve expuesto a entre 5 a 6 amenazas. Un $76 \%$ de las viviendas y un $87 \%$ de las empresas del país se encuentran en áreas 
con una o más amenazas naturales, ubicándose un $9.27 \%$ de las viviendas y $13 \%$ de los negocios en corregimientos con entre 5 y 6 amenazas. En cuanto a la red vial, un 59\% de la misma se encuentra en corregimientos afectados por al menos 1 amenaza, 3.75\% en áreas con entre 5 a 6 amenazas.

b) Índice de Riesgo Manifiesto: Este índice permite identificar los corregimientos con el mayor impacto debido a la combinación de un elevado número de personas, muertes y viviendas afectadas durante el período de estudio. De acuerdo a los resultados de este análisis, de los 10 corregimientos con mayor índice de riesgo manifiesto, al menos 5 se encontraban en la provincia de Panamá, 4 de ellos en el distrito capital. Juan Díaz, Tocumen y Pacora, en el distrito de Panamá, y Cativá en Colón, son los corregimientos urbanos con los índices de riesgo manifiesto más altos. Estos corregimientos se caracterizan por una alta proporción tanto de viviendas construidas entre el 2006 y el 2010 (de entre el 5 y 34\% de todas las viviendas en estos corregimientos), como de población migrante llegada entre 2005 y 2010, (entre el 10 y el 22\% del total de la población).

Por su parte, el corregimiento de Guabito, en Changuinola, provincia de Bocas del Toro, presenta características de las áreas rurales del país, con una alta proporción de población indígena $(67 \%)$, dedicada a actividades agropecuarias (44\%), viviendas sin acceso a servicios de agua potable $(90 \%)$, electricidad $(36 \%)$ y saneamiento $(45 \%)$.

Estos 5 corregimientos con el índice de riesgo manifiesto más alto, también se caracterizan por su carácter multiamenazas, encontrándose todos entre 3 y 5 amenazas concurrentes. 
Invest. pens. crit.

Vol. 2, No. 5, septiembre-diciembre 2014.

pp. 04-25

Figura 5. Mapas de Índice de Riesgo Manifiesto (IRM) y de Amenazas Naturales según corregimientos.

Panamá: 1990- 2013.

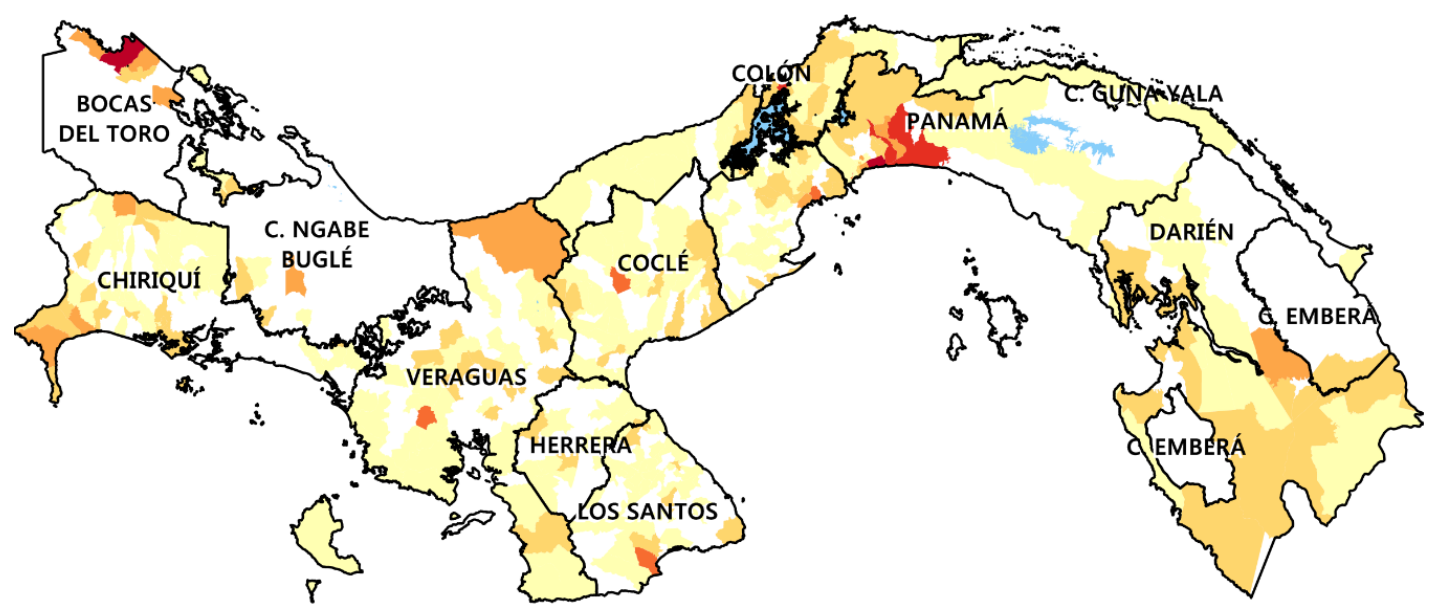
IRM
$<0$
$1 \ldots 2$
$2.1 \ldots 4.0$
$4.1 \ldots 7.0$
$7.1 \ldots 10.0$
$>10.0$

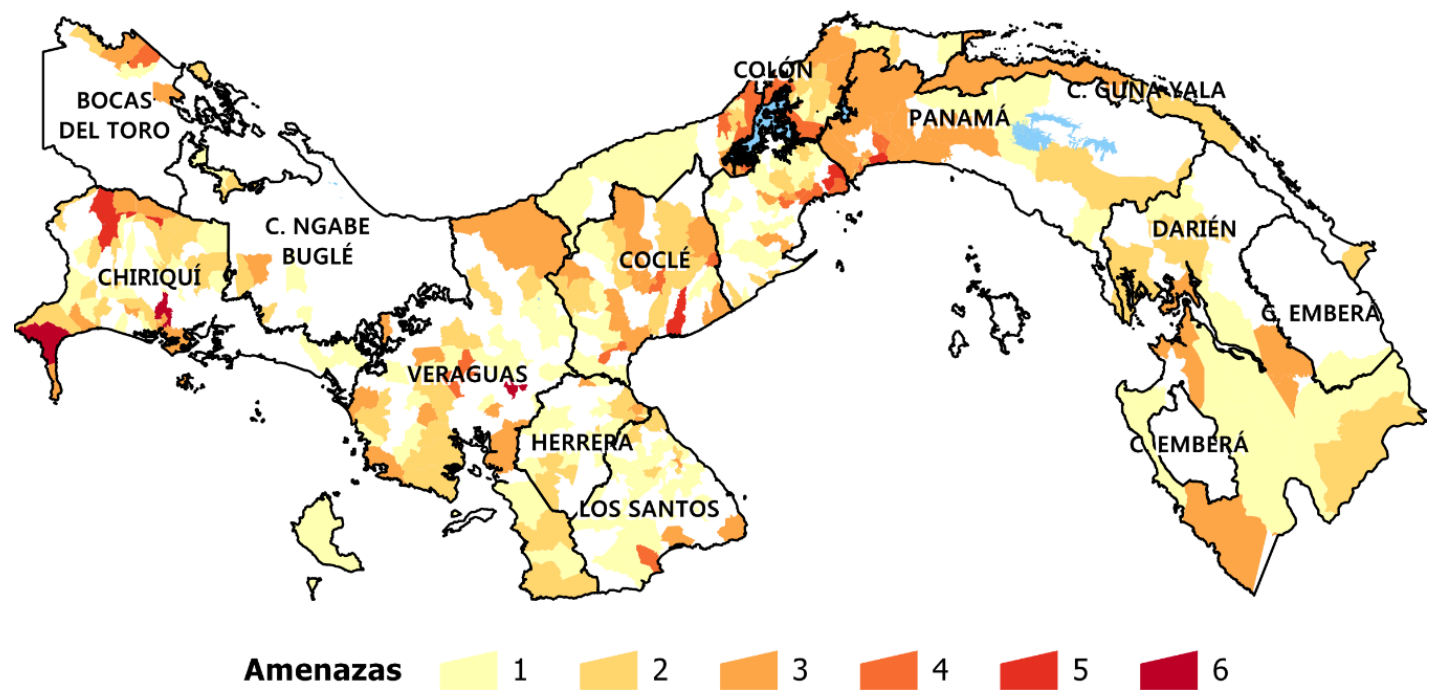

Fuente: elaborado por el autor a partir de la base de datos de Desinventar.

\section{$\underline{\text { Impactos económicos de los desastres naturales }}$}

Dada las debilidades de la base de datos Desinventar, en cuanto al registro de los impactos en sectores de infraestructura y económicos, fue necesaria la revisión de Gacetas Oficiales y medios de prensa en la internet, con el fin de estimar el impacto económico de los desastres de mayor envergadura ocurridos en Panamá en el período 2004- 2014. 
Tabla 3. Eventos e impacto económico de los principales desastres registrados en Panamá entre 2004 y 2014.

\begin{tabular}{|c|c|}
\hline Evento & Impactos \\
\hline $\begin{array}{l}\text { Inundación en la cuenca del } \\
\text { Río Juan Díaz, Panamá, } 2004\end{array}$ & $\begin{array}{l}\text { Recuperación de daños en viviendas por US } 2,73 \text { millones de dó- } \\
\text { lares. }\end{array}$ \\
\hline $\begin{array}{l}\text { Inundación en el poblado de } \\
\text { Guásimo, Colón (Cuenca del } \\
\text { Río Indio), } 2006\end{array}$ & $\begin{array}{l}\text { Se estima que la inversión para recuperar la infraestructura perdida } \\
\text { por estas inundaciones de unos US } 25 \text { millones de dólares. }\end{array}$ \\
\hline $\begin{array}{l}\text { Inundaciones Bocas del Toro, } \\
2008\end{array}$ & $\begin{array}{l}\text { La inversión para la reconstrucción de los tramos afectados por los } \\
\text { deslizamientos en este evento de la carretera Rambala- Gualaca se } \\
\text { estimó en US } 25 \text { millones de dólares. }\end{array}$ \\
\hline $\begin{array}{l}\text { Inundaciones en Costa Abajo } \\
\text { de Colón, Chepo y San Migue- } \\
\text { lito, } 2010 \text { (La Purísima): }\end{array}$ & $\begin{array}{l}\text { US 149,3 millones de dólares; daños en ganado, US 10,4 millones; } \\
\text { daños en la potabilizadora de Chilibre, } 10 \text { millones; US } 25 \text { millones } \\
\text { para el MIVIOT para reposición de viviendas afectadas; US 8,4 mi- } \\
\text { llones para } 18 \text { instalaciones de salud; } 103,8 \text { millones reparación de } \\
\text { la carretera de acceso a Puente Centenario. }\end{array}$ \\
\hline Sequía mayo- junio 2012 & $\begin{array}{l}\text { El fenómeno generó una reducción de la producción artesanal de } \\
\text { leche y además los pequeños productores de subsistencia se retra- } \\
\text { saron en el cultivo a chuzo de maíz y arroz. Fondos asignados por } \\
\text { el Gobierno de Panamá, US } 1 \text { millón de dólares. }\end{array}$ \\
\hline $\begin{array}{l}\text { Inundaciones en las provin- } \\
\text { cias de Colón, Coclé y Pa- } \\
\text { namá, } 2012\end{array}$ & $\begin{array}{l}\text { US } 123 \text { millones de dólares: al MIVIOT, } \$ 24 \text { millones, al Ministerio } \\
\text { de Salud } \$ 2.4 \text { millones, al MEDUCA, } \$ 8.1 \text { millones y al IDAAN } \\
\text { unos } \$ 799 \text { mil; el MOP por } \$ 83.7 \text { millones; el MIDA } \$ 2.5 \text { millones; } \\
\text { la Lotería Nacional de Beneficencia } \$ 1.3 \text { millón, el Ministerio de } \\
\text { Gobierno (Sinaproc) } \$ 188 \text { mil. Al menos US } 20 \text { millones debieron } \\
\text { pagar las aseguradoras por los daños ocasionados por las inunda- } \\
\text { ciones. }\end{array}$ \\
\hline $\begin{array}{l}\text { Sequía enero- abril 2013, Re- } \\
\text { gión Central de Panamá }\end{array}$ & $\begin{array}{l}\text { Disminución del nivel de los embalses del Canal y de las hidroeléc- } \\
\text { tricas del país, adopción de 'medidas preventivas' con el fin de op- } \\
\text { timizar el uso del agua y la energía eléctrica. Fondos asignados por } \\
\text { el Gobierno de Panamá, US } 3 \text { millones de dólares. }\end{array}$ \\
\hline $\begin{array}{l}\text { Inundaciones Las Nubes, } \\
\text { Volcán, Chiriquí, agosto, } 2014\end{array}$ & $\begin{array}{l}\text { Impactos en el sector agrícola por US } 350 \text { mil dólares ( } 20 \text { produc- } \\
\text { tores). Impactos globales de este desastre superan los US } 2 \text { millo- } \\
\text { nes de dólares. }\end{array}$ \\
\hline $\begin{array}{l}\text { Aumento del nivel del mar y } \\
\text { relocalización de comunida- } \\
\text { des del Archipiélago Guna } \\
\text { Yala (próximos años) }\end{array}$ & $\begin{array}{l}\text { Se han asignado US } 2.4 \text { millones de dólares para la relocalización } \\
\text { de dos comunidades. }\end{array}$ \\
\hline
\end{tabular}

A partir de esta revisión bibliográfica podemos estimar que durante los últimos 10 años, los desastres naturales en Panamá han tenido un impacto económico acumulado de al menos US $\$ 353.43$ millones, afectando sectores tan diversos como las viviendas, infraestructura vial, escuelas, centros de salud, cultivos entre otros. Las dos últimas inundaciones registradas en 2010 y 2012, requirieron que el Gobierno de Panamá destinará fondos para la reparación de daños, equivalente al 1.4 y $0.85 \%$ de los 
Invest. pens. crit.

Vol. 2, No. 5, septiembre-diciembre 2014.

pp. 04-25

presupuestos estatales para cada uno de esos años, lo que es un indicativo del grado de impacto que registran los desastres ocurridos en Panamá.

\section{Discusión}

La realización de este análisis ha permitido elaborar un perfil de país en lo que respecta a la ocurrencia e impactos de desastres naturales en Panamá durante los últimos 23 años. Este perfil describe un país con un alto grado de impactos por desastres naturales de origen hidrometereológicos, y en menor medida por sismos y tsunamis.

Aun cuando una alta proporción de los impactos en población y viviendas se concentra en las áreas urbanas del país, las áreas rurales e indígenas aún representan la parte más importante de las afectaciones registradas.

Esta dinámica en la ocurrencia e impacto de los desastres naturales, podría ser indicativo de la existencia de dos escenarios en la forma en que se manifiestan las amenazas naturales: primero, un escenario urbano, de acelerado y desordenado crecimiento económico, altamente concentrado en la Región Metropolitana de Panamá; un segundo escenario, de áreas rurales, pobres, con una elevada proporción de población indígena dedicada a actividades agropecuarias, concentrado en zonas específicas del país como, el distrito de Changuinola, en Bocas del Toro, el corregimiento de Soná en Veraguas y Yaviza en Darién. De especial interés sería el caso de Changuinola, el cual presenta una recurrencia de inundaciones con grandes afectaciones en viviendas y población durante los años 2008, 2009 y 2010.

Se puede desprender también de este análisis que hay zonas que se ven afectadas por cierto tipo de eventos con mayor frecuencia que el resto del país. Tal es el caso de los deslizamientos, cuyas afectaciones se concentran en los distritos de San Miguelito en Panamá, Bugaba en Chiriquí y Santa Fe en Veraguas, los tornados, evento cuya ocurrencia e impactos se concentra en los distritos de Panamá y San Miguelito, o los sismos, cuyos mayores impactos se distribuyen entre el occidente de Chiriquí y Bocas del Toro.

La revisión de los datos de Desinventar permitió identificar vacíos de información y debilidades en cuanto al registro de los datos, que deben ser corregidos, entre las que encontramos:

- Ausencia de ciertos eventos ocurridos, pero no registrados en la base de datos. Tal es el caso del sismo de Bocas del Toro de 1991 y las sequías de 2010 y 2012, eventos que por su importancia obligaron a la declaración de emergencia por parte del Gobierno de Panamá y a la movilización de recursos y personal hacia las zonas afectadas por estos eventos, dada la magnitud de los mismos.

- Necesidad de que se revisen los datos respecto a la ocurrencia de incendios forestales e inclusive se realicen coordinaciones que permitan la incorporación de datos que colecta la ANAM y el Cuerpo de Bomberos de Panamá sobre la ocurrencia de estos eventos a la base de datos Desinventar con el fin de robustecer este registro. 
- Una revisión en medios de comunicación en la internet, permitió identificar tormentas eléctricas en las que hubo registro de muertes, pero que no aparecen en la base de datos, por lo que sería útil poder realizar las coordinaciones con ETESA y los servicios de salud en las diferentes provincias del país, para que estas instituciones reporten la ocurrencia de estos eventos a SINAPROC, (Radio La Primerísima, 2008).

- Desarrollar metodologías que permitan mejorar la capacidad de SINAPROC para realizar estimaciones rápidas de los daños, lo cual ayudaría a mejorar el registro de las variables de impacto de los eventos (sectores afectados, pérdidas económicas, daños en cultivos, etc.), datos que actualmente no se incorporan a la base de datos. El abordaje complementario que de los impactos económicos se hace en este estudio, a partir de la revisión bibliográfica, ayuda a identificar y cuantificar los recursos asignados por el Gobierno de Panamá para la atención de grandes eventos, pero indudablemente, no logra estimar la totalidad de los recursos utilizados, muy especialmente en aquellos eventos menores, para los cuales no fue posible obtener información.

El tema de la mejora en la resolución espacial de la identificación de la ocurrencia e impactos de los desastres de origen natural es de suma importancia para la generación de mapas de amenazas e impacto más robustos y detallados. Ante la ausencia de estudios sistemáticos que permitan identificar las diversas amenazas a que se encuentra expuesto el territorio, el registro de eventos que se realiza a través de Desinventar constituye un valioso aporte al conocimiento sobre la gestión del riesgo en Panamá.

En la medida en que la referencia geográfica de los eventos sea más específica, será posible ir mejorando estimaciones sobre el riesgo, la exposición y vulnerabilidad ante desastres en el país, y con ellos se podrá contar con mejores insumos para la construcción de mecanismos más efectivos para ordenar el desarrollo y minimizar los impactos por desastres en Panamá.

\section{Conclusiones}

Los datos presentados en este análisis permiten afirmar que Panamá es un país con un alto grado de exposición e impactos ocasionados por desastres naturales, los cuales durante los últimos 23 años han afectado la vida de más de medio millón de personas, además de 100 mil viviendas y ocasionado daños económicos cuantificados en al menos US $\$ 353.43$ millones, (lo que equivale al 1.8\% del Presupuesto General del Estado para el 2015).

En un escenario en que los datos indican que la magnitud de los impactos se ha incrementado en los últimos doce años, producto tanto de un rápido y desordenado proceso de crecimiento económico y urbano, como debido a la intensificación de los eventos hidrometereológicos como respuesta al Cambio Climático, se hace obligatoria la necesidad de replantear nuestro modelo de desarrollo, incorporando mecanismos que hagan efectivos los procesos de gestión integral del riesgo de desastres, y dejando de lado la antigua percepción de país con un bajo nivel de impactos debido a los mismos. 
Invest. pens. crit.

Vol. 2, No. 5, septiembre-diciembre 2014.

pp. 04-25

Un elemento fundamental en esta dirección, es la promoción de los procesos de generación de conocimientos, información y datos que ayuden en la identificación de las amenazas, la vulnerabilidad y la cuantificación de los impactos por desastres naturales. Sin conocimiento, la elaboración de políticas, la construcción de mecanismos de ordenación efectiva y de mitigación o adaptación frente al Cambio Climático, y los retos del desarrollo económico y urbano, se verán continuamente comprometidos por la ocurrencia de eventos cada vez más catastróficos o por condiciones cada vez más caóticas de crecimiento urbano, económico y social.

\section{Referencias}

anam.gob.pa, (2014). Planes de Manejos de Cuencas Aprobados. [online] Available at: http://anam.gob.pa/index.php/planes-de-manejos-de-cuencas-aprobados [Accessed 7 Nov. 2014].

Autoridad Nacional del Ambiente (ANAM), (2008). Atlas de las Tierras Secas y Degradas de Panamá. [ONLINE] disponible. Panamá: Autoridad Nacional del Ambiente, pp.2- 28.

Autoridad Nacional del Ambiente (ANAM), (n.d.). Aumento de la Resiliencia al Cambio Climático y la Variabilidad Climática en el Arco Seco y la Cuenca del Canal de Panamá. Nota Conceptual. Borrador Final de la Propuesta de Financiamiento de Panamá para presentar al Fondo de Adaptación. Panamá: Autoridad Nacional del Ambiente.

Arcia, O. (2014). Proyectos inmobiliarios en áreas proclives a desastres. La Prensa. [online] Available at: http://www.prensa.com/impreso/panorama/proyectos-inmobiliarios-areas-proclives-desastres/419673 [Accessed 7 Nov. 2014].

Banco Interamericano de Desarrollo/ Evaluación de Riesgos Naturales (ERN), (2012). Aplicación de los indicadores de riesgo de desastre y de gestión de riesgos, como insumo del Plan de Evaluación del Programa. Evaluación del Índice de Gestión del Riesgo.. Panamá: Banco Interamericano de Desarrollo.

Camacho, E. and Víquez, V. (1994). Licuefacción y hundimientos costeros en el noroeste de Panamá durante el Terremoto de Limón. Rev. Geol. Amér. Central.

CATHALAC/USAID, (2008). Potential Impacts of Climate Change on Biodiversity: in Central America, Mexico, and Dominican Republic. Panamá: CATHALAC.

Comisión Económica para América Latina y el Caribe (CEPAL), D (2014). Estadisticas.cepal.org. Available at: http://estadisticas.cepal.org/cepalstat/WEB_CEPALSTAT/Portada.asp [Accessed 15 Jul. 2014]. 
Comisión Económica para América Latina y el Caribe (CEPAL), (2010). Panamá: Efectos del Cambio Climático sobre la agricultura. CEPAL/CCAD/DFID. México, México: Comisión Económica para América Latina y el Caribe (CEPAL), pp.10- 12.

Conades.gob.pa, (2014). [online] Available at: http://www.conades.gob.pa/Informes-y-Documentos [Accessed 7 Nov. 2014].

DARA/ AECID, (n.d.). Condiciones y capacidades para la reducción del riesgo. Selección de Unidades Territoriales Representativas. País piloto: Panamá. Panamá: DARA/ AECID, pp.12- 14.

Data.worldbank.org, (2014). World Development Indicators | Data. [online] Available at: http://data.worldbank.org/products/wdi [Accessed 12 Sep. 2014].

Desinventar.org, (2014). DesInventar Project - Official Website. [online] Available at: http://www.desinventar.org/es/database [Accessed 12 Jul. 2014].

Espinosa, J. (2014). Memorias del Segundo Simposio Internacional: Construyendo caminos de conocimiento para un futuro con sostenibilidad hídrica. In: Gestión del agua en el Canal de Panamá durante la inundación extrema en diciembre 2010. Panamá: ACP/ UNESCO, pp.53- 57.

Fernández A., M. (2011). Daños, efectos y amenaza de tsunamis en América Central. Rev. Geol. Amér. Central, (26).

Fernández, M.; Molina, E, Havson, J., Atakan, K., (2000). Tsunamis and Tsunami Hazards in Central America. Natural Hazards, [online] 22(2), pp.91-116. Available at: http://link.springer.com/article/10.1023/A:1008102600622\#page-1 [Accessed 13 Sep. 2013].

Sanahuja, H (1999): El daño y la evaluación del Riesgo en América Central: Una propuesta metodológica tomando como caso de estudio a Costa Rica. La Red de Estudios Sociales en Prevención de Desastres en América Latina. Tesis de Postgrado, Maestría en Geografía de la Universidad de Costa Rica, pp 66. Avaible at: http://www.desenredando.org/public/libros/1999/haris/EvaluacionRiesgoAmericaCentral-1.0.1.pdf. [Accessed 13 Sep. 2013].

Sanahuja, H, Coles, A, Froes, P (2011): Under a Veneer of Resilience: Panama's City growing disaster risk. International Federation of Red Cross and Red Crescent Societies (IFRC), pp 87- 95.

Instituto de Geociencias, Universidad de Panamá, (2013). Terremotos destructivos más importantes en Panamá. Año: 1600- Actualidad. [imagen] Available at: http://panamaigc-up.com/wp-content/uploads/2013/07/Territorio-Panama.png [Accessed 16 Nov. 2013].

Instituto Nacional de Estadística y Censo, (2012). Censo de Población y Vivienda 2010. Resultados Finales Básicos. Panamá: Instituto Nacional de Estadística y Censo. 
Invest. pens. crit.

Vol. 2, No. 5, septiembre-diciembre 2014.

pp. $04-25$

Instituto Nacional de Estadística y Censo, (2012). Estimación del Producto Interno Bruto provincial, según categoría de Actividad Económica, a precios de 1996: años 1996-2010. Cuentas Nacionales. Instituto Nacional de Estadística y Censo: Panamá, p.P5521.

Instituto Nacional de Estadística y Censo, (2011). Indicadores económicos. Año 2010 y I Semestre 2011. Situación Económica. Panamá: Instituto Nacional de Estadística y Censo.

Laboratorio de Ingeniería Sísmica. Instituto de Investigaciones en Geociencias. Universidad de Costa Rica, (2004). Sismos en la Penínusla de Burica entre diciembre del 2003 y febrero del 2004. San José, Costa Rica: Laboratorio de Ingeniería Sísmica. Instituto de Investigaciones en Geociencias. Universidad de Costa Rica.

Ministerio de Desarrollo Agropecuario, (2009). Plan Estratégico para Mitigar los efectos del fenómeno de El Niño en el sector agropecuario (2009-2010). Panamá: Ministerio de Desarrollo Agropecuario.

Radio La Primerísima, (2008). Panamá: zona de alta densidad de descargas eléctricas. [online] Available at: http://www.radiolaprimerisima.com/noticias/34832/panama-zona-de-alta-densidad-dedescargas-electricas [Accessed 8 Nov. 2014].

Rockwell, T., Gath, E., Gonzalez, T., Madden, C., Verdugo, D., Lippincott, C., Dawson, T., Owen, L., Fuchs, M., Cadena, A., Williams, P., Weldon, E. and Franceschi, P. (2010). Neotectonics and Paleoseismology of the Limon and Pedro Miguel Faults in Panama: Earthquake Hazard to the Panama Canal. Bulletin of the Seismological Society of America, 100(6), pp.3097-3129.

Seismo.ethz.ch, (1999). index. [online] Available at: http://www.seismo.ethz.ch/static/gshap/ [Accessed 15 Oct. 2013].

Sistema Nacional de Protección Civil, (2012). Informe Nacional de Progreso en la Implementación del Marco de Acción de Hyogo (2011 - 2013). Preliminar. Panamá: Prevention Web.

Sistema Nacional de Protección Civil, (2012). Diagnóstico de Necesidades en Desarrollo de Capacidades para implementar la PNGIRD. Panamá: Prevention web.

Soulas, J. (1991). El sismo de Limón-Changuinola (Costa Rica y Panamá) del 22 de abril de 1991. Informe Preliminar de Misión. Panamá: Comisión Nacional de Riesgos y Atención de Emergencias de Costa Rica, pp.1- 2.

The Peninsula Principles in Action, (2014). Climate Change and displacement in the Autonomus Region of Guna Yala. Mission Report. The Peninsula Principles in Action, p.01.

U.S. Geological Survey, (2008). , Volcan Baru-eruptive history and volcano-hazards assessment. U.S. Geological Survey. 
Invest. pens. crit.

Vol. 2, No. 5, septiembre-diciembre 2014.

pp. $04-25$

World Bank, (2005). : Natural Disaster Hotspots: A Global Risk Analysis. Disaster Risk Management Series. Washington, DC: World Bank, p.4.

World Bank/ Global Facility for Disaster Reduction and Recovery, (2012). Panamá. Disaster Risk Management in Central America: Country notes. World Bank/ Global Facility for Disaster Reduction and Recovery, p.4. 